\title{
The Importance of Mental Health Training for Psychologists in COVID-19 Outbreak
}

\author{
Seyedeh Fahimeh Shojaei ${ }^{1,{ }^{*}}$ and Roya Masoumi ${ }^{2}$ \\ ${ }^{1}$ Radiation Oncology Department, Firoozgar Hospital, Iran University of Medical ScienceS (IUMS), Tehran, Iran \\ ${ }^{2}$ Educational Psychology, IAUCTB, Tehran, Iran \\ "Corresponding author: MSc, Clinical Psychologist, Radiation Oncology Department, Firoozgar Hospital, Iran University of Medical ScienceS (IUMS), Tehran, Iran. Email: \\ shojaee.fahimeh@yahoo.com
}

Received 2020 March 18; Accepted 2020 March 25.

Keywords: Attending Psychologists, Mental Health Literacy, Coronavirus (COVID-19), Outbreak Crisis

In early December 2019, coronavirus disease 2019 (COVID-19) was identified including the symptoms of pneumonia of unknown origin in Wuhan, the capital city of Hui Province, China (1), and it is now widespread in many countries around the world. In addition to a high rate of mortality, the disease has caused severe anxiety and psychological problems in the communities involved (2). The World Health Organization (WHO) has recently declared the novel coronavirus (2019-nCoV) outbreak a public health (3). In such a situation, training mental health professionals should be widely considered. It has been revealed that half of the Iranian population has a limited health literacy, which is more common in vulnerable groups, such as the elderly, housewives, unemployed people, and those with lower education levels (4). Mental health literacy is an important factor in determining health, by which both individual and community health can be promoted (5).

Anxiety, fear, and stress are often present in patients with COVID-19 or those suspected to have. The family of these patients or the people they are in contact with will also have these psychological issues such psychological problems can very easily create mass hysteria. Also, epidemiologists, scientists, doctors, and all health professionals as health-care heroes can also be affected by these mental issues because of being involved in this global crisis and making incredible sacrifices.

Several studies have reported negative psychological complications, including symptoms of traumatic stress, confusion, and anger in cases involved in stressful situations. The long quarantine period, fear of infection, despair, boredom, inadequate food shortages, inadequate information, financial loss, and stigma are known as stres- sors in the COVID-19 epidemic (6-8). Therefore, to improve mental health in Iran, people should receive psychological services and be informed about the way to access these services through various means, including social media, telephone, and online services, or in person.

The Iranian Association of Psychology and the Ministry of Health are recommended to establish a task force for physicians, psychiatrists, psychologists, and social workers to conduct workshops and psychological training courses through social media. For example, they can develop a psychological protocol through the Association of Psychology (designed by experts in mental health and healthcare) according to the level of mental health literacy of individuals and provide it to psychologist members of the organization.

Educational concepts can also be utilized through social media campaigns to introduce educational information and special precautions. Through these means, physicians, psychotherapists, and patients can share their experiences of fear, anxiety, and concerns, and talk about the experiences and events related to their health and illness. People with a lower educational level may be at a greater risk of misunderstanding the information and take health care issues less seriously (9). These people are suggested to be educated using educational images. Makin phone calls can also be helpful for temporary psychological counseling (10).

The groups of people who may need psychological services should be recognized and classified to be communicated; for example:

1. Patients with COVID-19 (with mild to severe symptoms)

2. People who have tested positive for 2019-nCoV

Copyright (c) 2020, Middle East Journal of Rehabilitation and Health Studies. This is an open-access article distributed under the terms of the Creative Commons Attribution-NonCommercial 4.0 International License (http://creativecommons.org/licenses/by-nc/4.0/) which permits copy and redistribute the material just in noncommercial usages, provided the original work is properly cited. 
3. The patient's family, colleagues, and friends

4. Medical staff (all medical staff)

5. Researchers working in laboratories studying on 2019-nCoV

6. People living in areas at high risk of COVID-19

7. People taking care of their elderly at home

8. Mothers, especially those who have children aged 8 to 10 years

\section{Pregnant women}

10. Working children and marginalized people

11. The general public (with all types of jobs)

12. Patients with particular mental disorders (patients with obsessive-compulsive disorder and mysophobia)

13. Patients with psychiatric disorders who also need medication

Conclusion: The suggestions provided in this study can provide an insight into the psychological impacts of COVID-19 as an emerging disease. Psychological services can play an important role at any stage to develop a more comprehensive and balanced plan to reduce future social and psychological stresses. Lack of efficient and proper professional support can lead to fatigue, despair, and helplessness among all members of the society, and it is worth noting that there will be a second wave of psychological health problems following the economic crisis caused by this outbreak, such as unemployment, falling prices in stock markets, etc. Such solutions and suggestions are effective to ensure no delay in medical and psychological care so that the crisis cannot lead to irreparable harm to society.

However, due to the limited number of studies available, more studies should be conducted on the psychological impacts of the COVID-19 outbreak. These studies are recommended to focus on the individual and group evaluations and interventions, training courses for medical staff, public education, responsive communication, updating new information about psychological health education, and professional implementation of the mental health educational protocols for people at risk.

\section{Footnotes}

Authors' Contribution: Seyedeh Fahimeh Shojaei designed the study, collected the data, and drafted the manuscript. Roya Masoumi revised the manuscript and reviewed scientific papers. All the authors read and approved the final manuscript.

Conflict of Interests: The authors declared no conflict of interests.

Ethical Approval: All procedures on human subjects were conducted based on the ethical standards of the Insti- tutional and/or National Research Committee and the 1964 Declaration of Helsinki and its later amendments, or comparable ethical standards.

Funding/Support: This study was not supported by any government or academic bodies.

\section{References}

1. Huang C, Wang Y, Li X, Ren L, Zhao J, Hu Y, et al. Clinical features of patients infected with 2019 novel coronavirus in Wuhan, China. Lancet. 2020;395(10223):497-506. doi: 10.1016/S0140-6736(20)30183-5. [PubMed: 31986264].

2. Liu S, Yang L, Zhang C, Xiang YT, Liu Z, Hu S, et al. Online mental health services in China during the COVID-19 outbreak. Lancet Psychiatry. 2020;7(4):e17-8. doi: 10.1016/S2215-0366(20)30077-8. [PubMed: 32085841].

3. World Health Organization. Coronavirus disease (COVID-19) outbreak. 2020. Available from: https://www.who.int.

4. Montazeri A, Tavousi M, Rakhshani F, Azin SA, Jahangiri K, Ebadi M, et al. Health Literacy for Iranian Adults (HELIA): Development and psychometric properties. Payesh (Health Monitor). 2014;13(5):589-99.

5. Kelly CM, Jorm AF, Wright A. Improving mental health literacy as a strategy to facilitate early intervention for mental disorders. Med J Aust. 2007;187(S7):S26-30. [PubMed: 17908021].

6. Duan L, Zhu G. Psychological interventions for people affected by the COVID-19 epidemic. Lancet Psychiatry. 2020;7(4):300-2. doi: 10.1016/S2215-0366(20)30073-0. [PubMed:32085840].

7. Yang Y, Peng F, Wang R, Guan K, Jiang T, Xu G, et al. The deadly coronaviruses: The 2003 SARS pandemic and the 2020 novel coronavirus epidemic in China. J Autoimmun. 2020:102434. doi: 10.1016/j.jaut.2020.102434. [PubMed: 32143990].

8. Brooks SK, Webster RK, Smith LE, Woodland L, Wessely S, Greenberg $\mathrm{N}$, et al. The psychological impact of quarantine and how to reduce it: Rapid review of the evidence. Lancet. 2020;395(10227):912-20. doi: 10.1016/S0140-6736(20)30460-8. [PubMed: 32112714].

9. Brzycki M. Improving low health literacy in poststroke patients by educating the health care staff. Electronic Theses and Dissertations; 2020. Available from: https://digitalcommons.acu.edu/etd/184.

10. Chinese Society of Psychiatry. Expert consensus on managing pathway and coping strategies for patients with mental disorders during prevention and control of serious. Chin J Psychiatry. 2020;53. 